\title{
Electrospun Nanofibrous Meshes Cultured With Wharton's Jelly Stem Cell: An Alternative for Cartilage Regeneration, Without the Need of Growth Factors
}

\author{
Marta Alves da Silva, Albino Martins, Ana R. Costa-Pinto, Nélson Monteiro, \\ Susana Faria, Rui L. Reis, and Nuno M. Neves*
}

Many efforts are being directed worldwide to the treatment of OA-focal lesions. The majority of those efforts comprise either the refinement of surgical techniques or combinations of biomaterials with various autologous cells. Herein, we tested electrospun polycaprolactone (PCL) nanofibrous meshes for cartilage tissue engineering. For that, articular chondrocytes (hACs) isolated from human osteoarthritic joints and Wharton's Jelly Stem Cells (hWJSCs) are cultured on electrospun nanofiber meshes, without adding external growth factors. We observed higher glycosaminoglycans production and higher overexpression of cartilage-related genes from hWJSCs cultured with basal medium, when compared to hACs isolated from osteoarthritic joints. Moreover, the presence of sulfated proteoglycans and collagen type II is observed on both types of cell cultures. We believe that this effect is due to either the electrospun nanofibers topography or the intrinsic chondrogenic differentiation potential of hWJSCs. Therefore, we propose the electrospun nanofibrous scaffolds in combination with $\mathrm{hWJSCs}$ as a viable alternative to the commercial membranes used in autologous chondrogenic regeneration approaches.

\section{Introduction}

Articular cartilage pathological conditions still present great demands in the field of orthopaedic medicine. A wide variety of those conditions involve loss of articular cartilage, for example osteoarthritis (OA) or rheumatoid arthritis (RA). ${ }^{[1]}$ Furthermore,

Dr. M. Alves da Silva, Dr. A. Martins, Dr. A. R. Costa-Pinto,

Dr. N. Monteiro, Prof. R. L. Reis, Prof. N. M. Neves

3B's Research Group

Biomaterials, Biodegradables and Biomimetics, Dept. of Polymer Engineering, University of Minho

Headquarters of the European Institute of Excellence on Tissue Engineering and Regenerative Medicine, AvePark, 4806-909 Taipas,

Guimarães, Portugal

E-mail: nuno@dep.uminho.pt

Dr. M. Alves da Silva, Dr. A. Martins, Dr. A. R. Costa-Pinto,

Dr. N. Monteiro, Prof. R. L. Reis, Prof. N. M. Neves

ICVS/3B's Laboratório Associado PT Government Associate

Laboratory, Portugal

Prof. S. Faria

Department of Mathematics for Science and Technology, Research CMAT

University of Minho, Guimaraes, Portugal

DOI: 10.1002/biot.201700073 osteochondral or chondral lesions in the joint cartilage may evolve to OA. One of the main characteristics of OA lesions is an imbalance in the joint cartilage that causes remodelling of the subchondral bone and changes in the composition of the synovial membrane. ${ }^{[2]}$

Microfracture has been widely used as a one-step arthroscopic procedure, involving debridment and exposure of the subchondral bone, allowing bone marrow to flow into the synovial space. ${ }^{[3]}$ The major drawbacks of microfracture are the poor biomechanical properties of the repaired tissue, a partial defect filling, and abnormal bone growth at the lesion area. ${ }^{[4]}$ Autologous transplantation approaches are focused in repairing the articular cartilage by using implants that carry autologous cells, isolated from a biopsy and pre-cultured in vitro. ACI (autologous chondrocytes implantation) has become the most applied clinical cell based therapy for the repair of cartilage injuries over the past decade. In this technique, expanded autologous articular chondrocytes are implanted under a periosteal flap after surgical debridement of the lesion. ${ }^{[5]}$ ACI has demonstrated excellent short to mid-term repair although the evaluation of longterm repair remains somewhat controversial. ${ }^{[6]}$ The technique was further developed into the use of scaffolds as supports for cell growth (e.g., MACI - Matrix induced articular chondrocytes implantation), ${ }^{[7]}$ where other sources of autologous cells including mesenchymal stem cells (MSCs), periosteum-derived cells, and synovial fibroblasts ${ }^{[8]}$ may be used. However, both ACI and MACI involve two surgical interventions (one for removing healthy cells from the donor site, and the other to implant the cells/matrix), as well as several limitations concerning the patient mobility and quality of life, namely donor site morbidity, long-term pain, and reduced mobility. Moreover, the outcome of those approaches is suboptimal, since the tissue formed consists frequently of fibrocartilage, which is not fully functional, nor long lasting. ${ }^{[9]}$

The most common tissue source of adult MSCs is the bone marrow, ${ }^{[10,11]}$ and these cells (BMSCs) have been used inseveral Tissue Engineering (TE) applications, including for cartilage repair. ${ }^{[10,12-15]}$ Yet, issues such as the narrow number of available MSCs in bone marrow, ${ }^{[16]}$ and the likelihood of donor site morbidity leads to the interest in identifying other MSCs sources. Human umbilical cord Wharton's jelly has been 
reported as a prospective alternative tissue source, ${ }^{[17,18]}$ since human umbilical cord MSCs share many properties with BMSCs, thus supporting their suitability for cell-based therapies. ${ }^{[19]}$ The source tissue from where stem cells are isolated may influence the chondrogenic effect of co-culturing chondrocytes with those MSCs. ${ }^{[20]}$ In the case of WJSCs, their ability for osteogenic differentiation with the support of hierarchical scaffolds has been reported by our group. ${ }^{[21]}$ Moreover, their ability to undergo chondrogenic differentiation in a trachea tissue engineering approach has been recently reported. ${ }^{[22]}$ Some other works have been exploring the chondrogenic potential of WJSCs with or without a 3D support, ${ }^{[23-25]}$ but all of them use exogenous growth factor supplementation for the induction of the cartilage phenotype. However, the use of bioactive factors has raised several limitations: their half-life is too short to ensure in vivo efficacy, a single administration is usually not sufficient to attain a adequate biological effect, the quantities required are prohibitively expensive, and continuous protein production increases the likelihood of undesired outcomes. ${ }^{[26]}$ Moreover, human MSCs isolated from fetal and adult bone marrow have been reported to show distinct differences in their sensitivity to specific growth factors. ${ }^{[27]}$ Therefore, one may inquire if these growth factors can indeed trigger the coordinated sequence and level of signals required for MSCs differentiation pathways, without the loss of crucial genotypic and phenotypic functions. Employing a strategy that avoids the use of growth factors, such as the one being reported herein, will overcome the above mentioned limitations.

In this work we explore the prospective of electrospun PCL nanofiber meshes (NFMs) as an alternative to the conventional membranes supporting autologous transplantation approaches, such as Hyalograft or AMIC ${ }^{\mathbb{R}}$. These membranes are mainly based on hyaluronic acid or collagen, respectively. Electrospun NFMs used herein are produced using a synthetic polymer (PCL) that was already approved by the regulatory agencies for resorbable sutures and other implantable devices. Furthermore, our meshes do not involve animal-derived biomaterials such as collagen that usually pose concerns in the consistency and variability, as well as on probability of disease transmission. Electrospun PCL NFMs are intended to act as a selective barrier for cells present in the synovial fluid, but enabling permeation by the synovial fluid and soluble molecules. Along with this advantage, the PCL membranes present an architecture that recreates the fibrillar structure of the ECM. ${ }^{[28,29]}$ It is described that the nanoscale topography of materials can influence the fate of stem cells. ${ }^{[30]}$ Thus, the objective is to determine if the topography of the electrospun NFMs is also able, per se, to induce in vitro chondrogenic differentiation of human Wharton's Jelly Stem Cells (hWJSCs). We used human osteoarthritic articular chondrocytes (hACs) isolated from knee sample of patients undergoing arthroplasty surgeries, as clinically relevant controls.

\section{Experimental Section}

\subsection{Fabrication of Electrospun Meshes}

NFMs were fabricated by the electrospinning process, as previously described $^{[31]}$ and patent protected (WO 2014/016816). Briefly, a polymeric solution was prepared by dissolving PCL into an organic solvent mixture of chloroform:DMF (70:30), at a concentration of 17 wt- $\%$. The polymeric solution was put into a syringe with a coupled blunted metallic needle. The syringe was coupled to a syringe pump (model KDS100, KD Scientific, Holliston, MA) in order to control the flow rate of the solution. A high-voltage power supply $(0-25 \mathrm{kV})$ was applied to the needle to generate a constant electric field of $12 \mathrm{kV}$. The needle tip-to-collector distance and the flow rate were fixed at $20 \mathrm{~cm}$ and $1.0 \mathrm{~mL} \mathrm{~h}^{-1}$, respectively. The produced PCL nanofiber meshes presented a thickness between 40 and $60 \mu \mathrm{m}, 70-80 \%$ of porosity, determined by nCT (data not shown) and an average pore size of $2.7 \mu \mathrm{m}$. For more details on the PCL nanofiber meshes, please refer to Guimarães et al. ${ }^{[32]}$ After collection, nanofiber meshes were cut into $1 \mathrm{~cm}^{2}$ squares.

\subsection{Human Articular Chondrocytes Isolation}

Chondrocytes isolation was performed according to the method previously reported by us. ${ }^{[33]}$ Briefly, cells were isolated from human knees samples, namely from the medial and lateral condyles. Samples were collected after obtaining informed consent from the patients undergoing arthroplasties in Centro Hospitalar do Alto Ave, Guimarães, Portugal. Cartilage pieces were removed from the knee samples and went by sequential enzymatic digestion for hACs isolation. After cells isolation, they were plated and cultivated with expansion medium: Dulbecco's modified Eagle's medium (Sigma, D5671), containing $10 \mathrm{mM}$ Hepes buffer (Sigma, H0887), L-alanyl-L-glutamine (Sigma, G8541), Non-Essential Aminoacids (Sigma, M7145), antibiotic/ antimitotic solution (Gibco, 15240062), 10\% fetal bovine serum (Gibco, 10270106), and $10 \mathrm{ng} \mathrm{mL}^{-1}$ of basic Fibroblast Growth Factor (bFGF) (PeproTech, 100-18B). Cells were expanded until passage 4 and then used for seeding the NFMs.

\subsection{Human Wharton's Jelly Stem Cells Isolation}

Human umbilical cords were obtained after obtaining informed consent from full-term caesarian sections donors, under the scope of the cooperation agreement of the 3B́s Research Group of the University of Minho and the Obstetrics Department of the Hospital de São Marcos, Braga, Portugal. Human WJSCs were isolated according to the procedure previously described by us. $^{[33]}$ Briefly, cells were isolated from umbilical cords by enzymatic digestion, plated and cultured using $\alpha$-MEM medium (Gibco, 12000-063), supplemented with antibiotic/antimycotic solution and $10 \%$ fetal bovine serum. Cells were expanded until passage 4 and then seeded onto the NFMs.

\subsection{Cell Seeding Onto Electrospun PCL NFMs}

A suspension of $200000 \mathrm{hACs}$, or $200000 \mathrm{hWJSCs}$, was used for seeding the NFMs, in static culture conditions, at $37^{\circ} \mathrm{C}$ and $5 \%$ $\mathrm{CO} 2$, during $24 \mathrm{~h}$. The seeding density has been selected based on our previous reports using PCL NFMs. ${ }^{[31,34,35]}$ Basic medium was used: Dulbecco's modified Eagle's Medium containing $10 \mathrm{mM}$ Hepes solution, L-alanyl-L-glutamine, Non-Essential 
Aminoacids, antibiotic/antimicotic and 10\% fetal bovine serum. Afterwards, the constructs were transferred to new culture plates containing differentiation medium, in the case of hACs, or $\alpha$ MEM medium, in the case of hWJSCs. The hACs differentiation medium is a modification of the expansion medium: instead of adding bFGF, the medium was supplemented with $1 \mathrm{mg} \mathrm{mL}^{-1}$ of L-ascorbic acid (Sigma, A8960) and $50 \mathrm{mg} \mathrm{mL}^{-1}$ of insulin (Sigma, I5500). The $\alpha$-MEM medium was prepared as described above. The constructs were cultured during 28 days and the culture medium was changed every $2-3$ days.

\subsection{Cells Viability Assay}

Three constructs were collected at every time point: 7, 14, 21, and 28 days, and per each cell type, three per condition. A solution of $10 \%$ alamar blue (AbD Serotec, BUF012B) in differentiation medium (for hACs) or in $\alpha$-MEM (for hWJSCs) was added to each construct and incubated for $4 \mathrm{~h}$. After incubation, a $250 \mu \mathrm{L}$ aliquot was taken from each well and placed in a 96 well plate, in triplicate for each sample. OD was determined in a microplate reader (Bio-Tek, model Synergie HT, USA), at 570 and $600 \mathrm{~nm}$.

\subsection{Cells Proliferation Assay}

The evaluation of the cells' proliferation was performed using the Quant-IT PicoGreen dsDNA Assay Kit (Invitrogen, P7589), according to the manufacturer protocol. Triplicate samples were collected at each time point, for each type of cells, and a standard curve was established using known DNA concentrations $\left(0-1.5 \mu \mathrm{g} \mathrm{mL}^{-1}\right)$. Fluorescence of both the samples and of the standard curve concentrations was read with an excitation of $485 \mathrm{~nm}$ and an emission of $528 \mathrm{~nm}$, in a microplate reader (BioTek, model Synergie HT). The DNA concentrations of the samples were interpolated directly from the standard curve.

\subsection{DMB Assay for GAGs Quantification}

DMB assay was performed according to the method described by Kafienah and Sims. ${ }^{[36]}$ Briefly, three samples were collected at every time point, and for each cell type, frozen overnight and then digested. The digestion solution was prepared by adding $1 \mathrm{mg} \mathrm{mL}^{-1}$ of proteinase K (Sigma, P8044) diluted in $50 \mathrm{mM}$ Tris- $\mathrm{HCl}$, at $\mathrm{pH}$ of 7.6. Samples were incubated with $1 \mathrm{~mL}$ of the proteinase $\mathrm{K}$ solution overnight at $60^{\circ} \mathrm{C}$. For each assay, $20 \mu \mathrm{L}$ of samples and $250 \mu \mathrm{L}$ of DMB solution were used. A total of $20 \mu \mathrm{L}$ of water were added to a 96 well plate, as a blank. The plates were incubated for $10 \mathrm{~min}$ and then the optical density was measured in a microplate reader (Bio-Tek, model Synergie $\mathrm{HT})$, at $525 \mathrm{~nm}$.

\subsection{Total RNA Isolation}

Specimens were gathered at every time point (three per time point and per cell type), washed in PBS, immersed in Tri reagent (Sigma, T9424) and kept at $-80^{\circ} \mathrm{C}$ for posterior RNA extraction. The specimens were taken from the freezer and thawed for the RNA extraction procedure. Chloroform (Sigma, C2432) was added; the specimens were vigorously agitated for $15 \mathrm{~s}$ and then incubated in ice for $15 \mathrm{~min}$. After that incubation, the specimens were centrifuged at $13000 \mathrm{rpm}$, for $15 \mathrm{~min}$, at $4{ }^{\circ} \mathrm{C}$. Afterwards, the supernatant was collected for a sterile $1.5 \mathrm{~mL}$ tube, and an equivalent volume of isopropanol (VWR, 437423R) was added. The specimens were incubated at $-20^{\circ} \mathrm{C}$ overnight, to precipitate the RNA. The next day, the specimens were centrifuged at $13000 \mathrm{rpm}$, for $15 \mathrm{~min}$, at $4{ }^{\circ} \mathrm{C}$. Then, the supernatant was taken and $800 \mu \mathrm{L}$ of ethanol $70 \%$ was added to wash away the isopropanol. Tubes were agitated vigorously and centrifuged again, at $9000 \mathrm{rpm}$ for $5 \mathrm{~min}$, at $4{ }^{\circ} \mathrm{C}$. The supernatant was again removed, and the pellet was left to air dry. Finally, the pellet was re-suspended in $20 \mu \mathrm{L}$ of distilled water DNase, RNase free (Lonza, 733-1631). The concentration and purity of the extracted RNA was evaluated using the NanoDrop ND-1000 Spectrophotometer (NanoDrop Technologies Inc, USA).

\subsection{Quantitative Real-Time Polymerase Chain Reaction (qRT-PCR)}

This procedure consisted of a two-step fluorogenic assay using the PerfeCta ${ }^{\mathrm{TM}} \mathrm{SYBR}^{\circledR}$ Green system (Quanta Biosciences, 95055-100). All reagents used in this procedure were purchased from Quanta Biosciences, following the instructions of the manufacturer. Thermocycler reaction conditions used performed in accordance to the protocols suggested in the kit. In the first step, RNA was reverse transcribed into cDNA, using the qScript cDNA Synthesis Kit (95047-500). A MasterCycler EP Gradient detection system (Eppendorf, USA) was used to perform the reaction. Afterwards, the obtained cDNA was used as template for the amplification of the target genes shown in Table 1, with the PerfeCta ${ }^{\mathrm{TM}} \mathrm{SYBR}^{\circledR}$ Green FastMix $^{\text {TM }}$ kit (95072-05K). Tested genes include Aggrecan, Collagen Type I, Collagen Type II, COMP, and Sox9. GAPDH was used as house-keeping gene, and the expression of all target genes was normalized against the GAPDH of the same specimen for each time point of the study. All primer sequences were generated using Primer3 software (http:// biotools.umassmed.edu/bioapps/primer3_www.cgi) and acquired from MWG Biotech AG, Germany. Table 1 shows the details of the primers used.

\subsection{Morphological Observation of the Constructs}

For SEM analysis, samples were collected at 7 and 28 days of experiment. They were washed in sterile PBS and immersed in 3\% glutaraldehyde (Sigma/G-5882) at room temperature for $1 \mathrm{~h}$. Samples were again washed in PBS, dehydrated in increasing ethanol concentrations and let to dry. Samples were sputter coated with gold and analyzed. A scanning electron microscope (JSM-6010LV, JEOL, Japan) was used to observe cells distribution and morphologies. 
Table 1. Primers used for RT-PCR.

\begin{tabular}{|c|c|c|c|c|}
\hline Name & $\begin{array}{l}\text { Primer sequence }\left(5^{\prime}-3^{\prime}\right) \\
\text { forward/reverse }\end{array}$ & $\begin{array}{l}\mathrm{Tm} \\
\left({ }^{\circ} \mathrm{C}\right)\end{array}$ & $\begin{array}{l}\text { Product } \\
\text { size (bp) }\end{array}$ & $\begin{array}{l}\text { Sequence ref. } \\
\text { (NCBI) }\end{array}$ \\
\hline \multirow[t]{2}{*}{ GAPDH } & AGCCTCAAGATCATCAGCAA & 56.3 & 101 & NM_002046.4 \\
\hline & GTCATCAGTCCTTCCACGAT & & & \\
\hline \multirow[t]{2}{*}{ ACAN } & TGAGTCCTCAAGCCTCCTGT & 58.3 & 129 & NM_013227.3 \\
\hline & TGGTCTGCAGCAGTTGATTC & & & \\
\hline \multirow[t]{2}{*}{ COMP } & AGGGATGGAGACGGACATCAG & 58.0 & 340 & NG_007070.1 \\
\hline & TCTGCATCAAAGTCGTCCTC & & & \\
\hline \multirow[t]{2}{*}{ COL la } & AACAACCCCAAGGACAAGAG & 58.4 & 159 & NM_000088.3 \\
\hline & GTAGGTCATCTTCTGGGACG & & & \\
\hline \multirow[t]{2}{*}{ COL IIa } & GTTCACGTACACTCСССTCA & 59.4 & 156 & NM_001844.4 \\
\hline & TCCACACCGAATTCCTCCTC & & & \\
\hline \multirow[t]{2}{*}{ soxg } & TTCATGAACATCACCGACGC & 57.9 & 311 & NM_000346.3 \\
\hline & GTCCAGTCGTAGCCCTTCAG & & & \\
\hline
\end{tabular}

\subsection{Histological Analysis}

Samples were collected at the end of the experiment, fixed in $10 \%$ neutral buffered formalin and then kept at $4{ }^{\circ} \mathrm{C}$ until the staining procedures. For Alcian Blue staining, samples were rinse in 3\% acetic acid and kept in 1\% alcian blue solution (Sigma A-3157) for $1 \mathrm{~h}$. After that, stain was dispensed and sections were washed with water, let to dry and then rinsed in absolute alcohol, cleared in xylene and mounted in Entellan rapid (VWR, 1.07960.0500).

\subsection{Immunolocalization of Collagen Type II}

Immunolocalization of collagen type II was performed in fixed samples. Constructs were permeabilized with $0.2 \%$ Triton X100
(Sigma, X100) in PBS for $5 \mathrm{~min}$ and then it was blocked with a solution of 3\% BSA (Sigma, A2153) in PBS for $30 \mathrm{~min}$. Endogenous peroxidase activity was quenched with $0.3 \%$ hydrogen peroxide solution during $30 \mathrm{~min}$. Samples were rinsed in PBS during $5 \mathrm{~min}$. R.T.U. Vectastain ${ }^{\circledR}$ Universal Elite ABC Kit (Vector/VCPK-7200) was used for antibody incubation, according to the instructions of the manufacturer. Shortly, samples were incubated with anti-human collagen type II (Millipore Iberica/ MAB1330) overnight at $4{ }^{\circ} \mathrm{C}$, in a humidified atmosphere. Incubation was revealed by using the Peroxidase Substrate Kit DAB (Vector/VCSK-4100), according to the instructions of the manufacturer. Samples were washed in water during $5 \mathrm{~min}$ and counterstained with hematoxilin for nuclei visualization. Finally, slides were mounted in Entellan rapid.

\subsection{Statistical Analysis}

Data were statistically analyzed using IBM SPSS software (version 21; SPSS Inc.). The Shapiro-Wilk test was firstly applied to test the assumption of normality and the results showed that the data was not following a normal distribution. $p$ values lower than 0.05 were considered statistically significant.

\section{Results}

\subsection{Cells Viability and Proliferation}

Cell viability and proliferation was assessed during the all experiment (Figure 1A and B, respectively). HACs showed a significantly higher cell viability than hWJSCs in almost all time points of the experiment, except for the 21st day, where no significant difference between hACs and hWJSCs was found (Mann-Whitney $U$ test, $p<0$ 005) (Figure 1A). Cell proliferation results showed no significant differences between hACs and hWJSCs for almost all the time points of the experiment, except
A

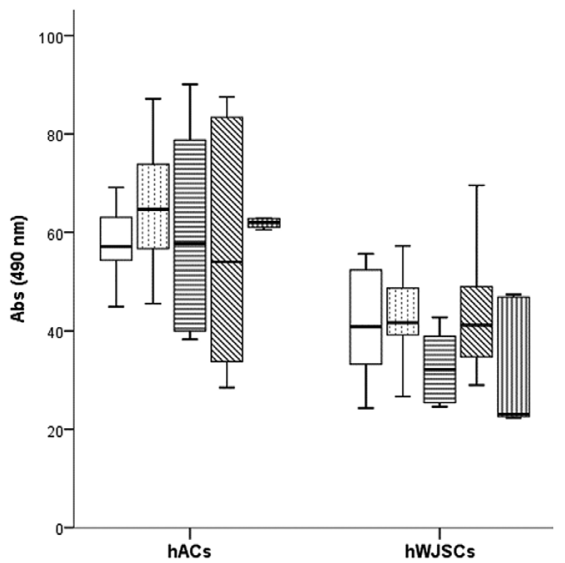

B

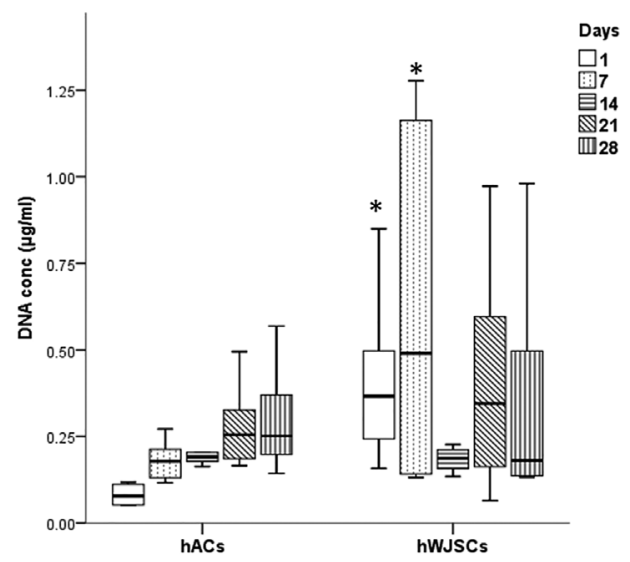

Figure 1. Box plots of cells viability and proliferation along the 28 days of the experiment. Data were analyzed by nonparametric way of a Mann-Whitney U-test. * Denotes significant differences compared to hACs cultures. A) Alamar blue test results for hACs and hWJSCs. B) Cell proliferation results (DNA concentration $\left[\mu \mathrm{g} \mathrm{mL}^{-1}\right]$ ) in hACs and hWJSCs. 
for the 1 st and 7th days, where hWJSCs displayed significantly higher cell proliferation values compared to hACs (MannWhitney $U$ test, $p<0.005)$, as stated in Figure 1B.

\subsection{GAGs Accumulation and Expression of Cartilage-Related Genes}

The GAGs content for each type of culture was assessed. Samples were collected at 7 and 28 days of the experiment
(Figure 2, insert). No significant difference between hACs and $\mathrm{hWJSCs}$ was found at 28 days of culture, but hWJSCs displayed a significantly higher GAGs concentration than hACs at 7 days of culture (Mann-Whitney $U$ test, $p<0.05$ ). Accordingly, several cartilage-related genes expression was also analyzed, and differences between the expressions in both types of cultures, on both time points (Figure 2) were determined. Cartilagerelated genes expression sustained the results obtained for GAGs accumulation in hWJSCs cultures, as Aggrecan, Collagen type II, and COMP expression was significantly higher in
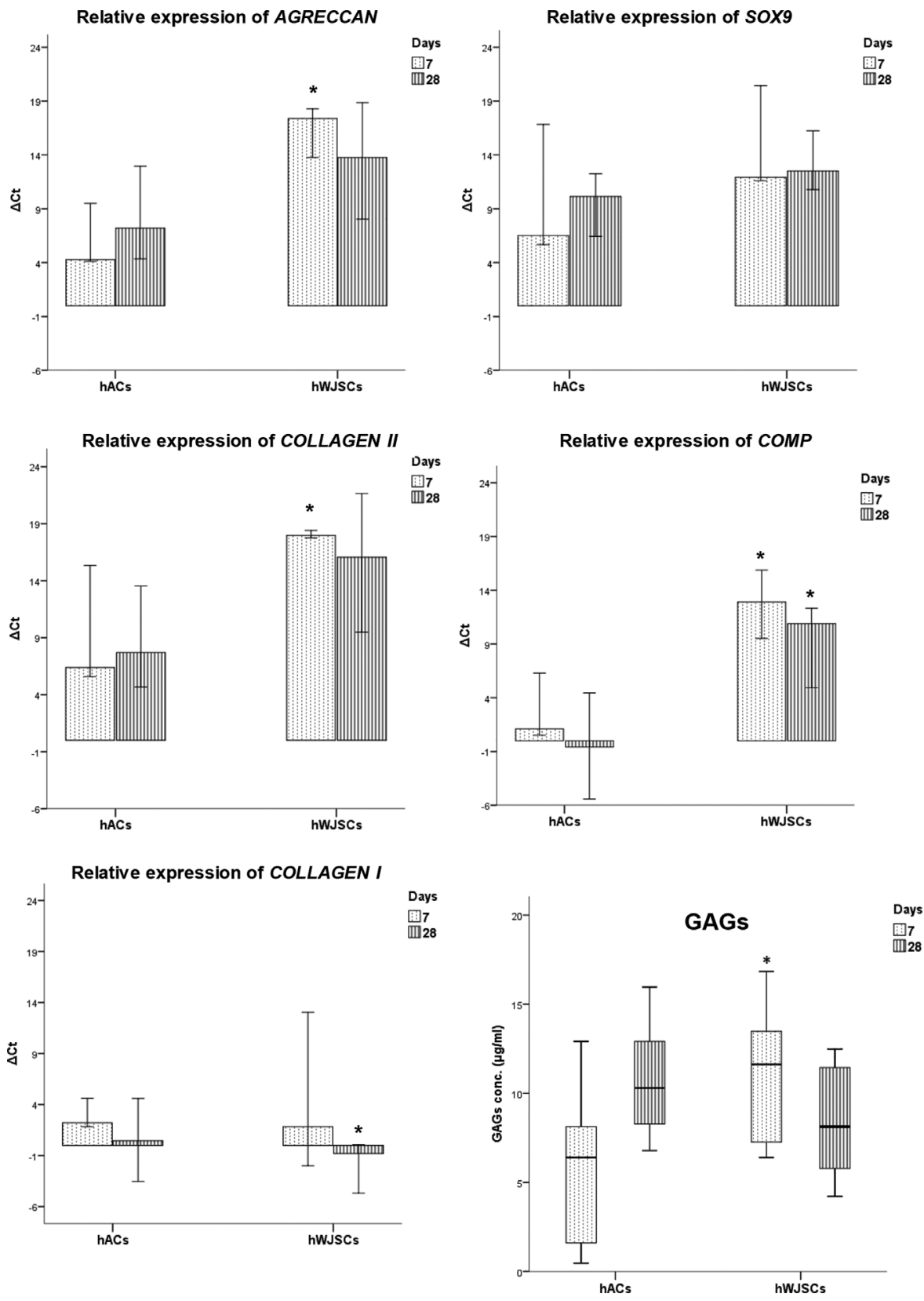

Figure 2. Bar plot of chondrogenic markers present in hACs and hWJSCs cultures, normalized for the reference gene GAPDH, after 7 and 28 days of culture. Data were analyzed by nonparametric way of a Mann-Whitney U-test. * Denotes significant differences compared to hACs cultures. Insert: Box plot of the GAGs concentration ( $\mu \mathrm{g} \mathrm{mL}^{-1}$ ) in hACs and hWJSCs after 7 and 28 days of culture. Data were analyzed by nonparametric way of a MannWhitney U-test. * Denotes significant differences compared to hACs cultures. 
hWJSCs compared to the hACs cultures. Moreover, Collagen type $I$ expression showed a significant decrease in those cultures when compared to hACs. In terms of COMP, hWJSCs displayed a significantly higher expression than hACs at 7 and 28 days. Aggrecan expression was significantly higher at 7 days between hWJSCs and hACs, but not at 28 days $(p=0.067)$ of experiment. SOX9 expression was not significantly different $(p=0.164$ at 7 days and $p=0.400$ at 28 days). No statistical differences were found for Collagen type II expression at 7 days of culture $(p=0.057)$, but at 28 days hWJSCs showed significantly higher values of this gene when compared to hACs $(p=0.042)$. Finally, concerning Collagen type I, no statistically significant expression was found for 7 days of culture $(p=0.057)$, but at 28 days hWJSCs showed significantly lower values when compared to hACs.

\subsection{Cells Distribution and Morphology}

Both types of cultures were analyzed by SEM to compare their morphology and distribution at the surface of the NFMs (Figure 3). Cells showed an even spreading along the PCL electrospun NFMs. Nevertheless, hACs seemed more dispersed than hWJSCs, which were clumped together. Concerning cells morphology, we can observe the typical chondrocyte round shape either for hACs (Figure 3A and insert) and hWJSCs, at 7 days of culture (Figure 3B and insert). There seems to be a loss of the chondrocyte phenotype at the end of the experiment for hACs (Figure 3C and insert), whereas for hWJSCs the morphology is maintained (Figure 3D and insert).

\subsection{Histological Results}

Alcian blue staining was performed in 28 days samples to identify sulfated proteoglycans present in cartilage matrix (Figure 4A-D), confirming the SEM observations. Staining in the NFMs seeded with hACs was spread along the NFMs, in the places cells were cultured. In the NFMs seeded with WJSCs, staining was localized in only one spot, where cells aggregated and formed cartilage-like ECM. Staining was more intense and localized, showing the proteoglycans production and accumulation.

Immunolocalization of collagen type II was also assessed as this protein is one of the major components of articular cartilage ECM (Figure 4E-H). Consistent with GAGs quantification and expression of cartilage-related genes, the histological analysis of hACs and hWJSCs cultures revealed sulfated proteoglycans deposition on both types of cultures (Figure 4A-D), as well as the existence of collagen type II in the engineered tissue formed (Figure 4E-H).

\section{Discussion}

Herein, we demonstrated the ability of hWJSCs chondrogenic differentiation when seeded onto electrospun PCL NFMs without growth factor induction. We have previously shown that PCL NFMs are suitable for cartilage TE approaches by supporting the growth, proliferation, and cartilagineous ECM deposition by bovine articular chondrocytes, either in static or in stirred conditions. ${ }^{[31]}$ Furthermore, electrospun PCL NFMs also support and promote human MSCs chondrogenic differentiation when using a flow perfusion bioreactor for their culture. ${ }^{[34]}$ Importantly,
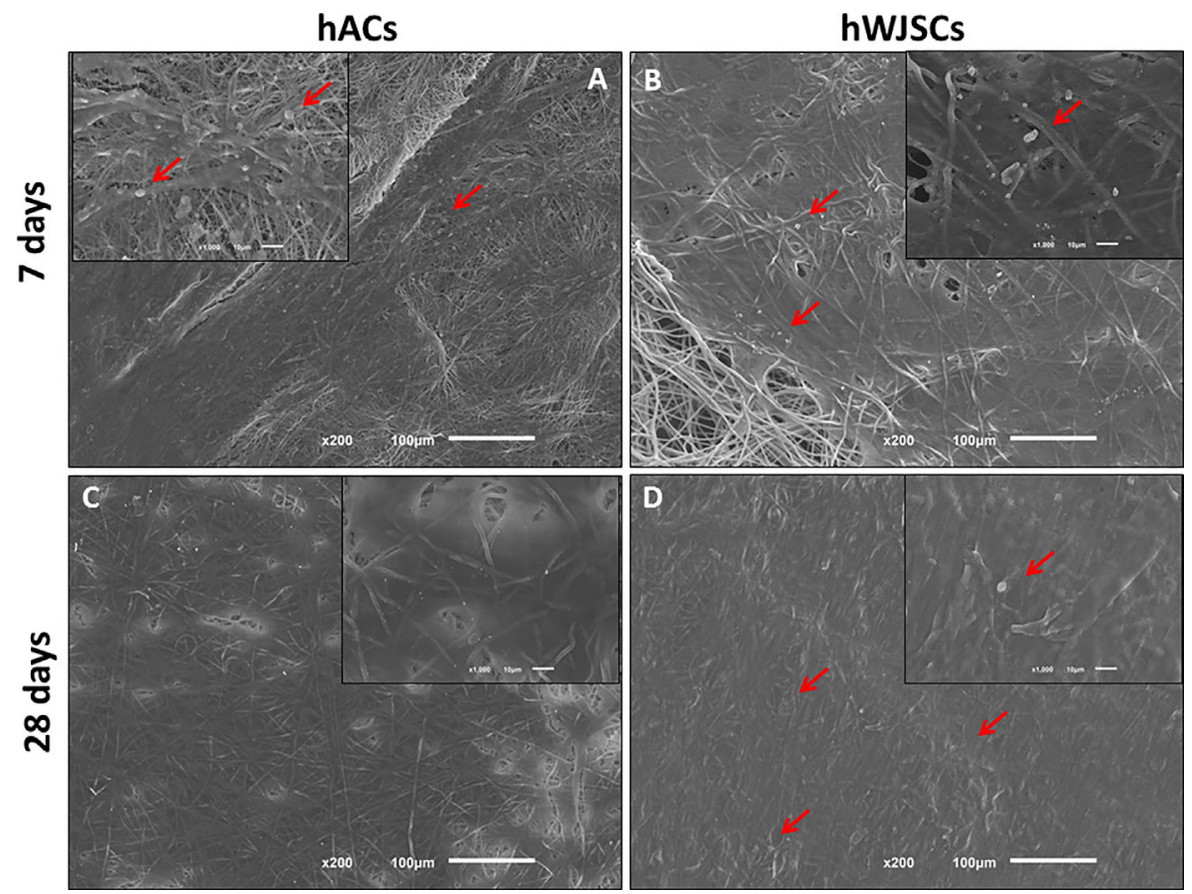

Figure 3. Scanning electron micrographs showing the hACs and hWJSCs distribution and morphology on the NFMs, at 7 (A, B) and 28 (C, D) days of experiment. Red dots show the round-shaped morphology of hACs. 
hACs
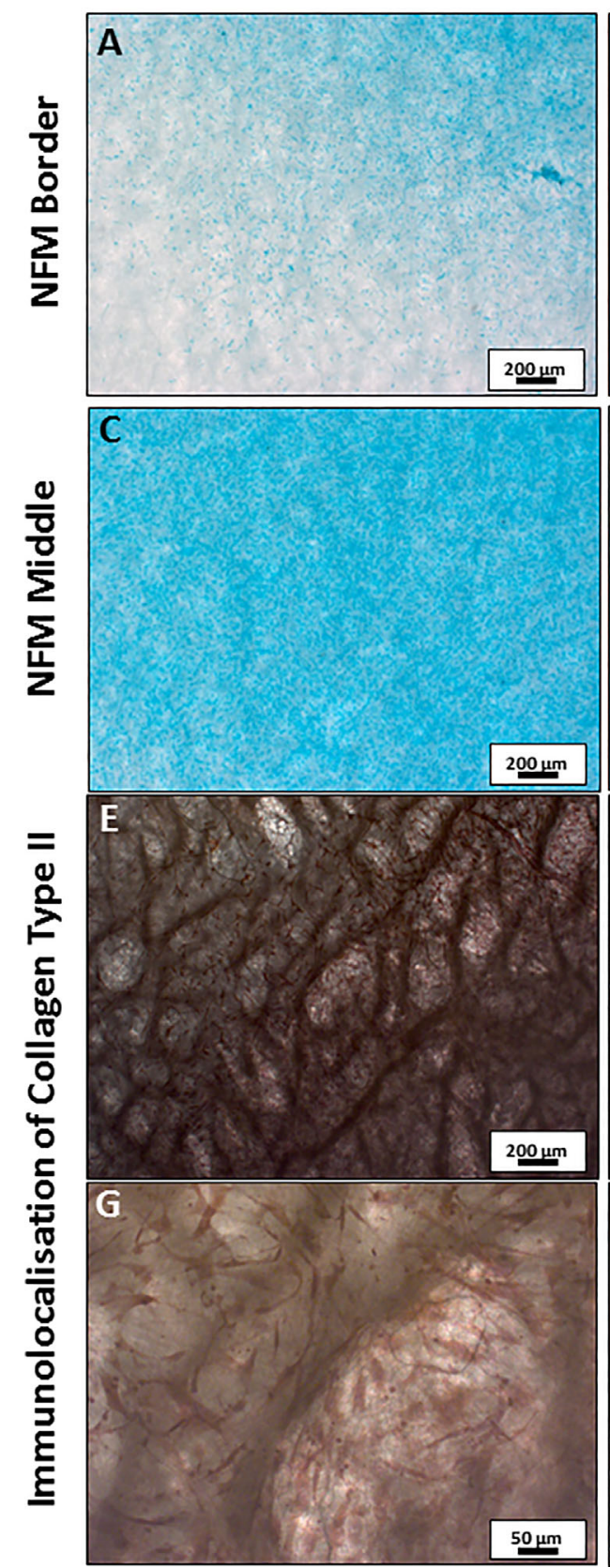

hWJSCs
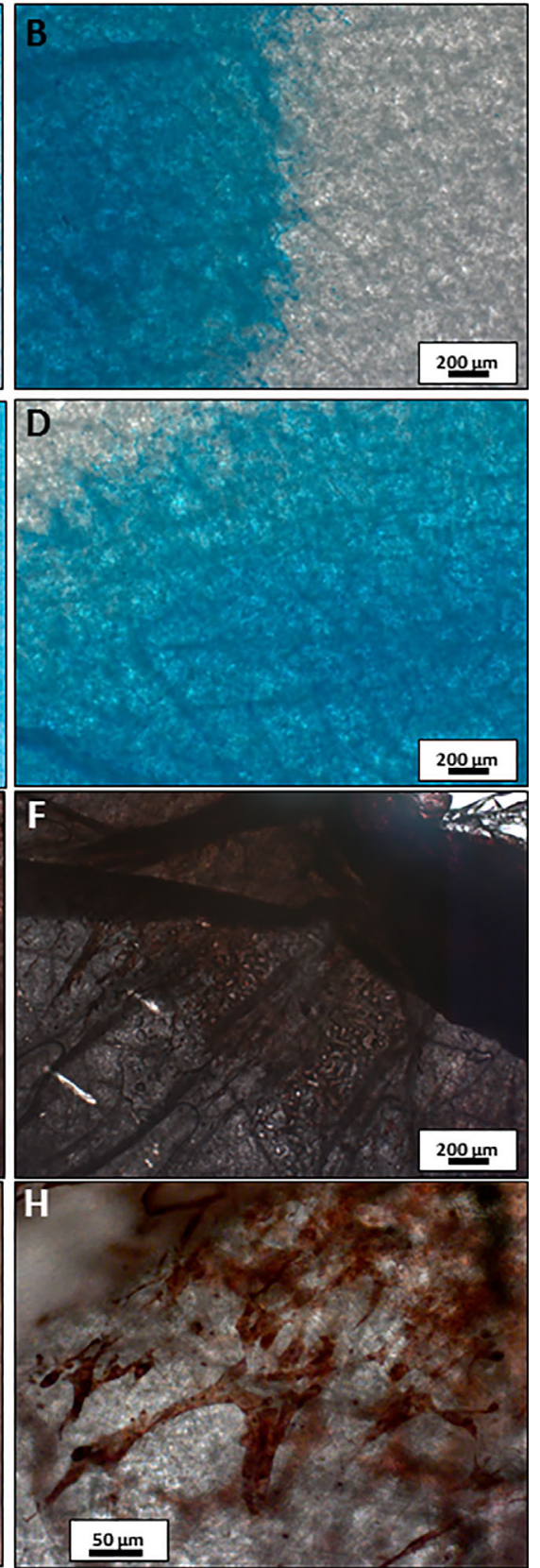

Figure 4. Histological analysis of hACs and hWJSCs cultures in PCL nanofiber meshes, at 28 days of culture. A-D) alcian blue staining. E-H) collagen type II immunolocalization.

we showed that PCL NFMs support the culture of both normal bovine chondrocytes, ${ }^{[31]}$ as well as human chondrocytes isolated from diseased joints. The maintenance of diseased hACs phenotype when seeded onto the NFMs is one fundamental aspect in our strategy. Although chondrocytes were isolated from the cartilage regions not directly affected, they were exposed to the synovial fluid affected by the pathological cartilage. It is speculated that electrospun PCL NMFs will be used for the treatment of main injuries of cartilage that can, in a further stage, develop into OA. Therefore, the use of these chondrocytes is important because we are testing the cells phenotype present at the implant site, as representative of a MACI technique with autologous cells.

Obtained results for cell viability and proliferation showed that hACs, although being significantly more metabolically active than hWJSCs (Figure 1A), have a proliferation rate significantly slower than hWJSCs (Figure 1B). Proliferation results confirm the enhanced proliferation rate of WJSCs reported in literature. ${ }^{[19]}$ Human ACs were able to produce GAGs when seeded onto the electrospun PCL NFMs, along the experiment (Figure 2, insert). Surprisingly, hWJSCs cultured in 
basal medium, without any induction of chondrogenic differentiation, presented a significantly higher value for GAGs production, when compared to hACs. Moreover, stem cells presented significantly higher expression of Aggrecan, COMP, and Collagen type II when equated with hACs (Figure 2). Moreover, the lower expression of Collagen Type I shows that the cartilage-like tissue formed is not fibrocartilage. This low expression indicated that the obtained ECM resembles the natural articular cartilage. These results are in accordance with the GAGs production, and show that hWJSCs could be driven in to the chondrogenic lineage by culturing in electrospun PCL NFMs using only basal media without any growth factors induction. One of the critical properties that can control cell in growth, proliferation within the scaffolds and integration with the surrounding tissues is the thee-dimensional porous structure of scaffolds. ${ }^{[37]}$ The pore structure also contributes to provide cells with the proper nutritional supply and spatial organization for tissue regeneration, ${ }^{[38]}$ as well as for cells phenotype development and stability in vitro. ${ }^{[39]}$ Both the proliferation and differentiation of MSCs can be positively influenced by the culture in 3D scaffolds. ${ }^{[40]}$ Moreover, that the material topography controls cell relations with materials, which determines their attachment, distribution, and cellular phenotype ${ }^{[41]}$ It was demonstrated in the literature that MSCs can be attracted into a cartilage defect by the direction of a collagenous matrix, after drilling a channel in the cartilage structure down to the subchondral bone. ${ }^{[42]}$ Therefore, we believe that the unique structure of the electrospun PCL NFMs ${ }^{[28,43]}$ may be providing cues for triggering stem cells differentiation. Indeed, we could observe by SEM that hACs could not maintain their typical round-shape morphology along the 28 days of culture, although the hWJSCs show this morphology already at the 7th day of culture (Figure 3). Moreover, these observations are sustained by the presence of sulfated proteoglycans on both types of cultures (Figure 4A-D) and also of collagen type II in both chondrocytes (Figure 4E and G) and hWJSCs (Figure 4F and H). Our results are in agreement with other reports, ${ }^{[44]}$ that have shown how the nanofibers architecture can control hBMSC fate. hBMSCs culture in nanofibers induced osteogenic differentiation without using osteogenic supplements, suggesting that the scaffold structure can be designed to guide cells into morphologies that direct their differentiation toward a chosen lineage ${ }^{[44]}$ Moreover, a recent report has described that biochemical (growth factors supplementation) and physical (nanofiber) cues regulated similar ontological pathways, suggesting an overlap in the molecular mechanisms that these stimuli use to control stem cell function. ${ }^{[45]}$ It has also been demonstrated that the structural properties of the extracellular or cell neighboring microenvironment regulate cell processes and signaling pathways. ${ }^{[39,40,45,46]}$

Human WJSCs have not been extensively explored for TE applications when compared to BMSCs, but present several competitive advantages, namely: 1) a higher frequency of colony forming units (CFUs-F), allowing obtaining a larger number of MSCs in the initial isolation procedure ${ }^{[17]}$;2) a higher proliferative potential ${ }^{[19]}$; 3) WJSCs represent an transitional stem cell type that partly combines some pluripotent properties of adult MSCs ${ }^{[4]}$; 4) extra-embryonic tissue-derived MSCs (such as WJSCs) have adjacent ontogenetic relationship with embryonic stem cells, which grants them immune privileged characteristics, and a broader multipotent plasticity ${ }^{[48]}$; 5) WJSCs can be used without major ethical problems as extra-embryonic tissues are normally rejected after birth ${ }^{[47,49]}$; 6) umbilical cords are easy to harvest and available in large numbers ${ }^{[19]}$ thus, they are good candidates for the autologous or allogeneic treatment of several diseases. ${ }^{[50]}$ Nevertheless, WJSCs are an allogeneic source when compared to hBMSCs, but their hypoimmunogenicity has been reported, ${ }^{[51]}$ and it has been shown that hWJSCs express crucial immunomodulatory molecules even when exposed to chondrogenic, osteogenic, or adipogenic differentiation in vitro. ${ }^{[52]}$

Results obtained herein allow us to conclude that we could benefit from the nanofibrous architecture of our electrospun NFMs and produce cartilage-like tissue without the addition of growth factors. Those observations represent an important progress beyond the available chondrocyte-based strategies using autologous fetal stem cells regeneration of focal cartilage lesions. By combining both electrospun NFMs structure and hWJSCs commitment, we can define a long term strategy for the use of these meshes in autologous transplantation approaches, as they will improve the intrinsic repair activity of focal cartilage lesions and the surrounding osteoarthritic tissue.

\section{Abbreviations}

COMP, Cartilage Oligomeric Protein; DMB, Dimethylmethylene Blue; DMF, Dimethylformamide; ECM, Extracellular Matrix; GAGs, Glycosaminoglycans; hACs, Human articular chondrocytes; hWJSCs, Human Wharton's Jelly Stem Cells; NFMs, Nanofiber meshes; PCL, polycaprolactone; qRT-PCR, quantitative real-time polymerase chain reaction.

\section{Acknowledgements}

The authors thank the Portuguese Foundation for Science and Technology for the Post-Doc grant of Marta Alves da Silva (SFRH/ BPD/73322/2010, financed by POPH - QREN - Tipologia 4.1 Advanced Formation, co-financed by Fundo Social Europeu and MEC national funds). This work was supported by the project SPARTAN (PTDC/CTM-BIO/4388/2014) FCT/MEC with PIDDAC funds. It was also partly supported by the POLARIS (FP7-REGPOT-2012-2013-1) and the Project "New methodologies for the isolation and control of stem cells differentiation using advanced culturing conditions and/or nanomaterials" (RL2 - SCN - NORTE-01-0124-FEDER-000018), co-financed by North Portugal Regional Operational Programme (ON.2 - O Novo Norte), under the National Strategic Reference Framework (NSRF), through the European Regional Development Fund (ERDF).

\section{Conflict of Interest}

The authors declare no financial or commercial conflict of interest.

\section{Keywords}

cartilage regeneration, chondrogenic differentiation, co-cultures, electrospinning, stem cells

Received: May 9, 2017

Revised: August 28, 2017

Published online: 
[1] A. P. Hollander, S. C. Dickinson, W. Kafienah, Stem Cells 2010, 28, 1992.

[2] J. Ringe, M. Sittinger, Arthritis. Res. Ther. 2009, 11, 211.

[3] J. R. Steadman, W. G. Rodkey, J. J. Rodrigo, Clin. Orthop. Relat. Res. 2001, (391 Suppl), S362.

[4] M. R. Steinwachs, T. Guggi, P. C. Kreuz, Injury 2008, 39, S26.

[5] M. Brittberg, A. Lindahl, A. Nilsson, C. Ohlsson, O. Isaksson, L. Peterson, N. Engl. J. Med. 1994, 331, 889.

[6] L. Batty, S. Dance, S. Bajaj, B. Cole, Anz. J. Surg. 2011, 81, 18.

[7] S. Marlovits, G. Striessnig, F. Kutscha-Lissberg, C. Resinger, S. M. Aldrian, V. Vecsei, S. Trattnig, Knee Surg. Sports Traumatol. Arthrosc. 2005, 13, 451.

[8] G. Schulze-Tanzil, Ann. Anat. 2009, 191, 325.

[9] P. Behrens, T. Bitter, B. Kurz, M. Russlies, Knee 2006, 13, 194.

[10] I. Sekiya, B. Larson, J. R. Smith, R. Pochampally, J.-O. Cui, D. J. Prockop, Stem Cells 2002, 6, 530.

[11] L. Wang, I. Tran, K. Seshareddy, M. L. Weiss, M. S. Detamore, Tissue Eng. Part A 2009, 15, 2259.

[12] D. Bosnakovski, M. Mizuno, G. Kim, S. Takagi, M. Okumura, T. Fujinaga, Cell Tissue Res. 2005, 319, 243.

[13] R. L. Mauck, X. Yuan, R. S. Tuan, Osteoarthritis Cartilage 2006, 14, 179.

[14] J. Hu, K. Feng, X. Liu, P. X. Ma, Biomaterials 2009, 30, 5061.

[15] B. D. Markway, G. K. Tan, G. Brooke, J. E. Hudson, J. J. Cooper-White, M. R. Doran, Cell Transplant. 2010, 19, 29.

[16] G. Chamberlain, J. Fox, B. Ashton, J. Middleton, Stem Cells 2007, 25, 2739.

[17] R. Sarugaser, D. Lickorish, D. Baksh, M. M. Hosseini, J. E. Davies, Stem Cells 2005, 23, 220

[18] H. S. Wang, S. C. Hung, S. T. Peng, C. C. Huang, H. M. Wei, Y. J. Guo, Y. S. Fu, M. C. Lai, C. C. Chen, Stem Cells 2004, 22, 1330.

[19] D. Baksh, R. Yao, R. S. Tuan, Stem Cells 2007, 25, 1384.

[20] F. Hildner, S. Concaro, A. Peterbauer, S. Wolbank, M. Danzer, A. Lindahl, P. Gatenholm, H. Redl, M. van Griensven, Tissue Eng. Part A 2009, 15, 3961.

[21] A. Canha-Gouveia, A. Rita Costa-Pinto, A. M. Martins, N. A. Silva, S. Faria, R. A. Sousa, A. J. Salgado, N. Sousa, R. L. Reis, N. M. Neves, Biofabrication 2015, 7, 035009.

[22] J. Wang, B. Sun, L. Tian, X. He, Q. Gao, T. Wu, S. Ramakrishna, J. Zheng, X. Mo, Mater. Sci. Eng. C Mater. Biol. Appl. 2017, 70, 637.

[23] E. Aleksander-Konert, P. Paduszynski, A. Zajdel, Z. Dzierzewicz, A. Wilczok, Cell Mol. Biol. Lett. 2016, 21, 11.

[24] P. Tanthaisong, S. Imsoonthornruksa, A. Ngernsoungnern, P. Ngernsoungnern, M. Ketudat-Cairns, R. Parnpai, PLoS ONE 2017, 12, e0168059.

[25] L. P. Merlin Rajesh Lal, G. K. Suraishkumar, P. D. Nair, J. Biomed. Mater. Res. A 2017, 105, 1845.

[26] J. E. Babensee, L. V. McIntire, A. G. Mikos, Pharmaceut. Res. 2000, 17, 497.
[27] K. Brady, S. C. Dickinson, P. V. Guillot, J. Polak, A. W. Blom, W. Kafienah, A. P. Hollander, Stem Cells Dev. 2013, 23, 541.

[28] A. Martins, J. V. Araujo, R. L. Reis, N. M. Neves, Nanomed 2007, 2, 929.

[29] A. Martins, R. L. Reis, N. M. Neves, Int. Mater. Rev. 2008, 53, 257.

[30] V. L. Lapointe, A. T. Fernandes, N. C. Bell, F. Stellacci, M. M. Stevens, Adv. Healthc. Mater. 2013, 2, 1644.

[31] M. L. Alves da Silva, A. Crawford, J. Mundy, A. Martins, J. V. Araujo, P. V. Hatton, R. L. Reis, N. M. Neves, Tissue Eng. Part A 2009, 15, 377.

[32] A. Guimaraes, A. Martins, E. D. Pinho, S. Faria, R. L. Reis, N. M. Neves, Nanomedicine (Lond) 2010, 5, 539.

[33] M. L. Alves da Silva, A. R. Costa-Pinto, A. Martins, V. M. Correlo P. Sol, M. Bhattacharya, S. Faria, R. L. Reis, N. M. Neves, J. Tissue Eng. Regen. Med. 2015, 9, 714.

[34] M. L. Alves da Silva, A. Martins, A. R. Costa-Pinto, P. Costa, S. Faria M. Gomes, R. L. Reis, N. M. Neves, Biomacromolecules 2010, 11, 3228 .

[35] A. Martins, A. R. Duarte, S. Faria, A. P. Marques, R. L. Reis, N. M. Neves, Biomaterials 2010, 31, 5875 .

[36] W. Kafienah, T. Sims, (Eds.), Biopolymer Methods in Tissue Engineering, Humana Press, NUS, Totowa, NJ 2004.

[37] R. Bagherzadeh, M. Latifi, L. Kong, J. Biomed. Mater. Res. Part A 2014 102A, 903.

[38] Q. P. Pham, U. Sharma, A. G. Mikos, Biomacromolecules 2006, 7, 2796.

[39] Y. Wang, U. J. Kim, D. J. Blasioli, H. J. Kim, D. L. Kaplan, Biomaterials 2005, 26, 7082

[40] A. C. Bean, R. S. Tuan, Biomed. Mater. 2015, 10, 015018.

[41] J. Jiang, E. T. Papoutsakis, Adv. Healthc. Mater. 2013, 2, 25.

[42] J. Kramer, F. Bohrnsen, U. Lindner, P. Behrens, P. Schlenke, J. Rohwedel, Cell Mol. Life Sci. 2006, 63, 616.

[43] A. Martins, E. D. Pinho, S. Faria, I. Pashkuleva, A. P. Marques, R. L. Reis, N. M. Neves, Small 2009, 5, 1195.

[44] G. Kumar, C. K. Tison, K. Chatterjee, P. S. Pine, J. H. McDaniel, M. L. Salit, M. F. Young, C. G. Simon, Biomaterials 2011, 32, 9188.

[45] B. A. Baker, P. S. Pine, K. Chatterjee, G. Kumar, N. J. Lin, J. H. McDaniel, M. L. Salit, C. G. Simon, Biomaterials 2014, 35, 6716

[46] W. T. Liu, Y. Wei, X. H. Zhang, M. M. Xu, X. P. Yang, X. L. Deng, Acs Nano 2013, 7, 6928.

[47] D. W. Kim, M. Staples, K. Shinozuka, P. Pantcheva, S. D. Kang, C. V. Borlongan, Int. J. Mol. Sci. 2013, 14, 11692.

[48] S. J. Prasanna, D. Gopalakrishnan, S. R. Shankar, A. B. Vasandan, PLOS ONE 2010, 5, e901.

[49] A. J. Marcus, D. Woodbury, J. Cell Mol. Med. 2008, 12, 730.

[50] A. Bongso, C. Y. Fong, Stem Cell Rev. Rep. 2013, 9, 226.

[51] C. Y. Fong, A. Subramanian, K. Gauthaman, J. Venugopal, A. Biswas, S. Ramakrishna, A. Bongso, Stem Cell Rev. Rep. 2012, 8, 195.

[52] G. La Rocca, M. Lo Iacono, T. Corsello, S. Corrao, F. Farina, R. Anzalone, Curr. Stem Cell Res. T. 2013, 8, 100. 